\title{
Staphylococcus aureus mediastinitis following a skin infection in a non-immunocompromised patient: A case report
}

\author{
KONSTANTINOS MANTZOURANIS ${ }^{1}$, VASILIKI EPAMEINONDAS GEORGAKOPOULOU ${ }^{2}$, \\ DIMITRIOS MERMIGKIS ${ }^{1}$, CHRISTOS DAMASKOS ${ }^{3,4}$, NIKOLAOS GARMPIS ${ }^{4,5}$, PETROS PAPALEXIS $^{6,7}$, \\ AIKATERINI GKOUFA ${ }^{8}$, PANAGIOTIS DROSSOS ${ }^{7}$, SERAFEIM CHLAPOUTAKIS $^{9}$, ANNA GARMPI ${ }^{6}$, \\ PAGONA SKLAPANI ${ }^{10}$, NIKOLAOS TRAKAS ${ }^{11}$ and XANTHI TSIAFAKI ${ }^{1}$
}

${ }^{1}$ 1st Pulmonology Department Sismanogleio Hospital, 15126 Athens; ${ }^{2}$ Pulmonology Department,

${ }^{3}$ Renal Transplantation Unit, Laiko General Hospital, Medical School, National and Kapodistrian University of Athens,

11527 Athens; ${ }^{4}$ N.S. Christeas Laboratory of Experimental Surgery and Surgical Research; Medical School,

National and Kapodistrian University of Athens, 11527 Athens; ${ }^{5}$ Second Department of Propedeutic Surgery;

${ }^{6}$ First Department of Propedeutic Internal Medicine, Laiko General Hospital, Medical School, National and Kapodistrian

University of Athens, 11527 Athens; ${ }^{7}$ Department of Biomedical Sciences, University of West Attica, 12243 Athens;

${ }^{8}$ First Department of Internal Medicine, Laiko General Hospital, Medical School, National and Kapodistrian University

of Athens, 11527 Athens; ${ }^{9}$ Department of Thoracic Surgery, Agios Savvas Hospital, 11522 Athens;

${ }^{10}$ Department of Cytology, Mitera Hospital, 15123 Athens; ${ }^{11}$ Department of Biochemistry,

Sismanogleio Hospital, 15126 Athens, Greece

Received September 12, 2021; Accepted October 12, 2021

DOI: $10.3892 / b r .2021 .1480$

\begin{abstract}
Mediastinitis is a severe inflammation of the structures located in the mid-chest cavity. Three main causes of infective mediastinitis are traditionally recognized: Deep infection of a sternal wound following cardiothoracic surgery, perforation of the esophagus, and the descending necrotizing mediastinitis as a result of odontogenic, pharyngeal or cervical infections. Mediastinitis, as a complication of skin infection with hematogenous spread is infrequent. Methicillin-resistant Staphylococcus aureus (MRSA) is a gram-positive bacteria, and is responsible for numerous severe infections. MRSA mediastinitis is a rare infection and is typically associated with complications of sternotomy and retropharyngeal abscesses. Here, the second known case of mediastinitis of a hematogenous origin in a non-immunocompromised 41-year-old patient following primary skin infection, accompanied by sternal osteomyelitis, lung consolidation and pleural effusion is described; MRSA was the responsible pathogen. The clinical course was favorable after 6 weeks of antibiotics administration without drainage or surgical intervention.
\end{abstract}

Correspondence to: Dr Vasiliki Epameinondas Georgakopoulou, Pulmonology Department, Laiko General Hospital, Medical School, National and Kapodistrian University of Athens, 17 Agiou Thoma Street, 11527 Athens, Greece

E-mail: vaso_georgakopoulou@hotmail.com

Key words: mediastinitis, staphylococcal skin infection, osteomyelitis, methicillin-resistant Staphylococcus aureus

\section{Introduction}

Mediastinitis, which is an inflammation of the chest region between the lungs, develops mostly due to a deep wound infection of the sternum, pneumonia, perforation of the esophagus, or descending necrotizing mediastinitis resulting from ear-nose-throat infections (1). Very rarely, mediastinitis can occur from hematogenous spread, and is usually a complication of the health care received (2).

Microorganisms frequently implicated in mediastinal infections include anaerobes, Streptococcus species, Corynebacterium species and members of the family Enterobacteriaceae (3). Methicillin-resistant Staphylococcus aureus (MRSA) continues to emerge as a causative agent of serious infections, mostly skin and soft tissue infections $(4,5)$. MRSA is more frequently related to mediastinal infections following cardiothoracic surgery, intravenous drug use and retropharyngeal abscesses (3).

Few cases of MRSA mediastinitis have been described. Herein, the rare case of MRSA mediastinitis accompanied by pulmonary consolidation, pleural effusion and sternal osteomyelitis following facial skin infection in, an otherwise healthy, 41-year-old man is reported.

\section{Case report}

A 41-year-old male non-smoking patient presented to Sismanogleio Hospital with fever and anterior thoracic pain over the previous 7 days. He had a medical history of gastroesophageal reflux, and was not receiving any medication. The patient had no history of diabetes mellitus, intravenous drug abuse, chronic obstructive pulmonary disease or other 


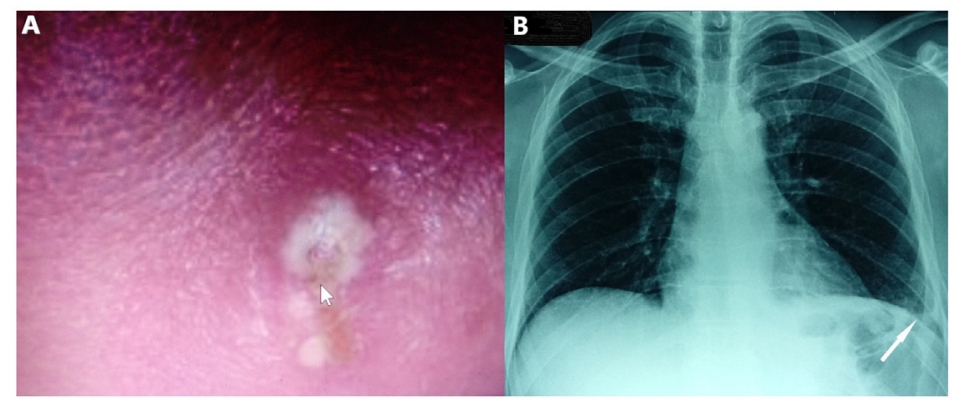

Figure 1. The skin lesion and chest X-ray on admission. (A) Pus-filled, $1 \mathrm{~cm}$ in greatest dimension, skin lesion on the patient's forehead. The skin over the infected area is red and swollen. (B) Chest X-ray shows consolidation in the left lower lobe with blunting of the left costophrenic angle.

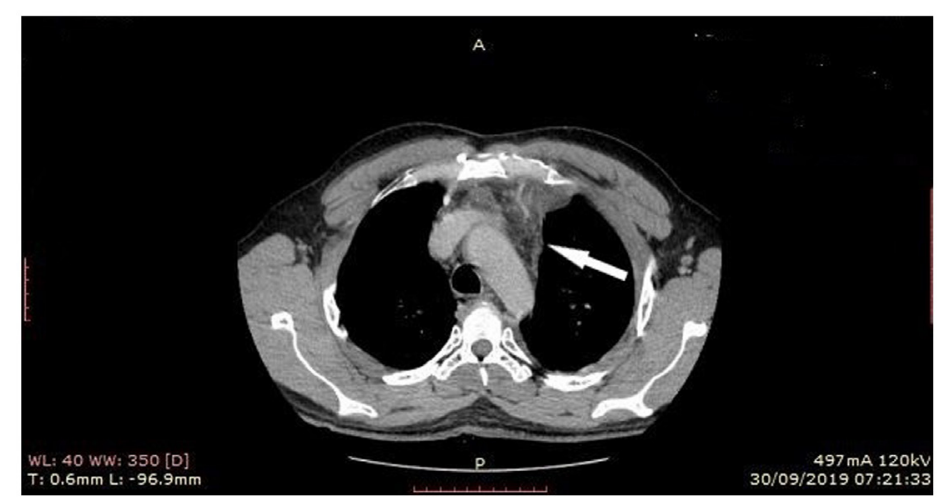

Figure 2. Chest computed tomography (mediastinal window) shows edema of the soft tissue adjacent to the first sternocostal joint and retrosternal fat heterogeneity/invasion. A, anterior; P, posterior.

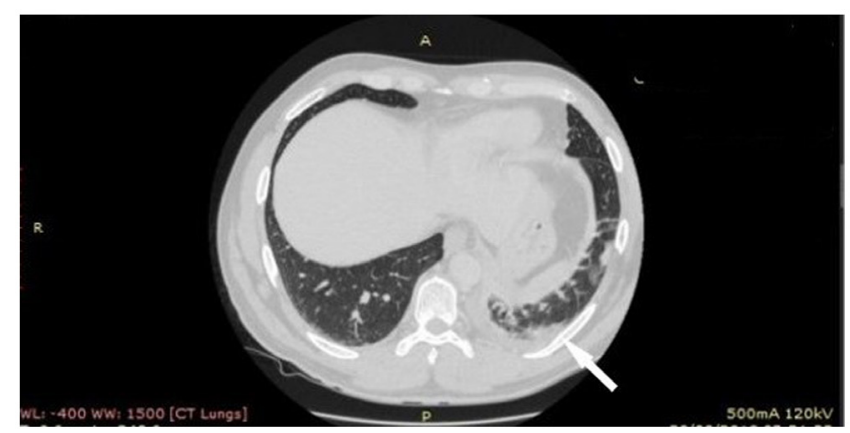

Figure 3. Lung window: Consolidation in the left lower lobe with left pleural effusion. A, anterior; P, posterior.

comorbidities, and no history of hospitalization and antibiotic intake in the previous 90 days.

Clinical evaluation revealed a febrile patient with dullness on percussion and crackles on auscultation at the base of the left lung. $\mathrm{He}$ also had boils and carbuncles in his face, with a pus-filled, $1 \mathrm{~cm}$ in greatest dimension, skin lesion on his forehead. The skin over the infected area was red and swollen (Fig. 1A). Physical examination of the oropharyngeal region did not reveal abnormal findings. Clinical examination did not also reveal neck lymph node enlargement or obvious inflammation of neck skin and soft tissues.

Blood pressure was $150 / 85 \mathrm{mmHg}$, heart rate was 89 beats per minute, oxygen saturation was $96 \%$ on room air and body temperature was $38.6^{\circ} \mathrm{C}$. Electrocardiography did not reveal abnormal findings on admission. Chest $\mathrm{X}$-ray revealed consolidation in the left lower lobe with blunting of the left costophrenic angle (Fig. 1B).
Laboratory investigation included complete blood cell count, and standard biochemistry serum and urine parameters. Laboratory findings included hemoglobin, $14.6 \mathrm{~g} / \mathrm{dl}$ (normal range, $12-15 \mathrm{~g} / \mathrm{dl}$ ); white blood cell count, $13.37 \times 10^{3} / \mu 1$ (normal range, $4-11 \times 10^{3} / \mu 1$ ); platelet count, $167 \times 10^{3} / \mu 1$ (normal range,

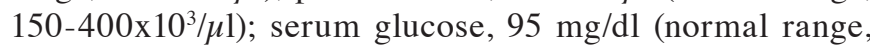
60-100 mg/dl), C-reactive protein (CRP), $96 \mathrm{mg} / \mathrm{l}$ (normal range, $<6 \mathrm{mg} / \mathrm{l}$ ) and D-dimers, $170.4 \mu \mathrm{g} / 1$ (normal range, $<500 \mu \mathrm{g} / \mathrm{l}$ ). Urinalysis and other blood biochemistry parameters were normal.

The patient underwent computed tomography (CT) of the chest showing consolidation in the left lower lobe with left pleural effusion, edema of the soft tissue adjacent to the first sternocostal joint and heterogeneity, and invasion of the retrosternal fat (Figs. 2 and 3). In addition, the patient underwent magnetic resonance imaging (MRI) of the chest, 
Table I. Prescription history and recovery course of the patient.

\begin{tabular}{ll}
\hline Day & \multicolumn{1}{c}{ Recovery course } \\
\hline Days 1-4 & Administration of intravenous Ceftriaxone, Vancomycin and Metronidazole \\
Day 2 & Improvement in clinical manifestations and blood inflammatory indices. \\
Day 4 & Fever subsidence. \\
Day 4 & Positive cultures from blood, pus and cystic lesions-isolation of Methicillin -resistant \\
& -resistant Staphylococcus aureus. \\
Day 4-28 & Administration of intravenous Vancomycin. \\
Days 29-42 & Administration of oral Ciprofloxacin . \\
Day 43 & Complete recovery; inflammatory indices within normal range.
\end{tabular}

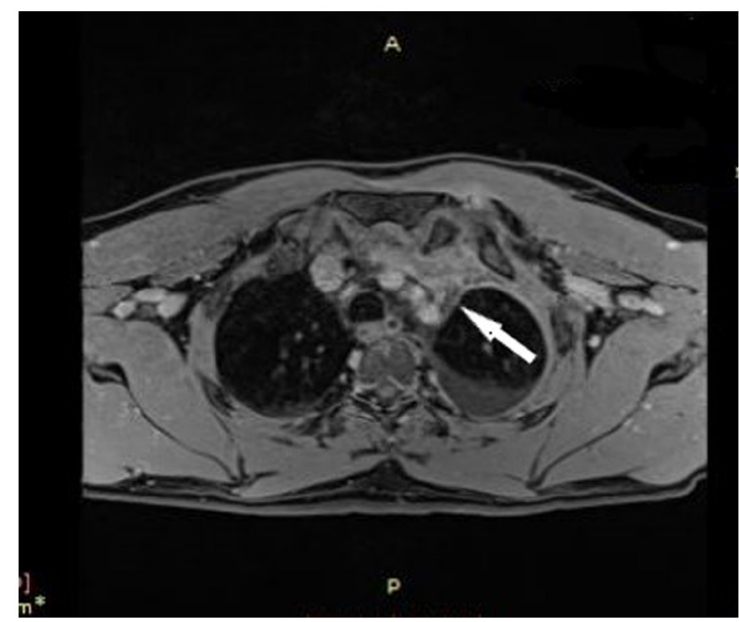

Figure 4. Magnetic Resonance Imaging of the chest shows a large abnormal soft tissue containing cystic lesions, adjacent to the first sternocostal joint. $\mathrm{A}$, anterior; $\mathrm{P}$, posterior.

to obtain detailed information regarding the pleura and mediastinum, that revealed abnormal soft tissue with dimensions at transverse level $7.0 \times 1.3 \mathrm{~cm}$, containing cystic lesions, adjacent to the first sternocostal joint, indicating inflammation in sternocostal cartilage. MRI also revealed contrast enhancement of the ipsilateral mediastinal pleura, imaging compatible with mediastinitis (Fig. 4).

Three blood cultures and culture of the pus from the area of facial infection were obtained before administration of antibiotics. The pleural effusion was assessed with pleural ultrasound and was not tapped due to its small size. The patient was treated with intravenous ceftriaxone ( $2 \mathrm{~g}$ single daily dose) and intravenous vancomycin (1 g twice daily) for pneumonia and skin infection, and metronidazole (500 $\mathrm{mg}$ three times daily) for anaerobic coverage regarding the suspected pleural infection empirically. MRSA, with susceptibility to ciprofloxacin, trimethoprim-sulfamethoxazole, vancomycin and linezolid, and resistance to clindamycin, was isolated both from blood cultures and from pus culture. MRSA was considered the causative agent for the mediastinal and lung lesions. A CT-guided aspiration of the cystic lesions for culture and susceptibility testing was performed, and this culture also revealed MRSA.

Transthoracic echocardiography was performed with normal ejection fraction and valve function. The patient was evaluated with an immunologic workup, with no immunodeficiency being identified. Immunoglobulin panel and $\mathrm{T}$ cell subpopulations were normal. Dihydrorhodamine testing for chronic granulomatous disease was also performed in order to exclude another rare etiology of severe MRSA infection. Human immunodeficiency virus testing was also negative.

The patient presented with improvement in clinical manifestations and blood inflammatory indices on the second day of hospitalization, and the fever subsided on the fourth day of hospitalization. There was no need for surgical procedure due to the good clinical response to antibiotics. The patient received intravenous vancomycin for 4 weeks and then there was a switch to oral treatment with ciprofloxacin $500 \mathrm{mg}$ twice daily for 2 weeks. Table I shows the prescription history and recovery flow of the patient. There was also complete recovery observed in the imaging evaluation (Fig. 5).

\section{Discussion}

The present report describes the rare case of a MRSA mediastinitis and sternal osteomyelitis infection accompanied by pulmonary consolidation and pleural effusion following skin infection, which was caused by a hematogenous spread in an immunocompetent host. Risk factors for MRSA infections include intravenous drug use, immunodeficiency, prior therapy with antibiotics and recent hospitalization. Young age, low socioeconomic status, and minority ethnicity have also been identified as emerging risk factors (3).

Moreover, MRSA mediastinitis is a particularly infrequent infection, and it has previously been known to be associated with complications of sternotomy and retropharyngeal abscesses (3). However, the patient described in the present case report did not have a retropharyngeal infection or cardiothoracic surgery, nor established immunodeficiency.

Common findings in mediastinitis are leukocytosis and elevations in CRP levels (6,7). In certain cases of acute mediastinitis, high levels of CRP are associated with a greater risk of death $(6,7)$. In the present case report, the patient also presented with increased levels of CRP. This biomarker was used to detect response to therapy, and it was within the normal range at the end of the treatment.

Although mediastinitis, as a result of hematogenous spread is rare, it has been described previously. Smith and Sinzobahamvya (8) reported an interesting case of an 11-year-old child with septic arthritis due to Staphylococcus aureus, 


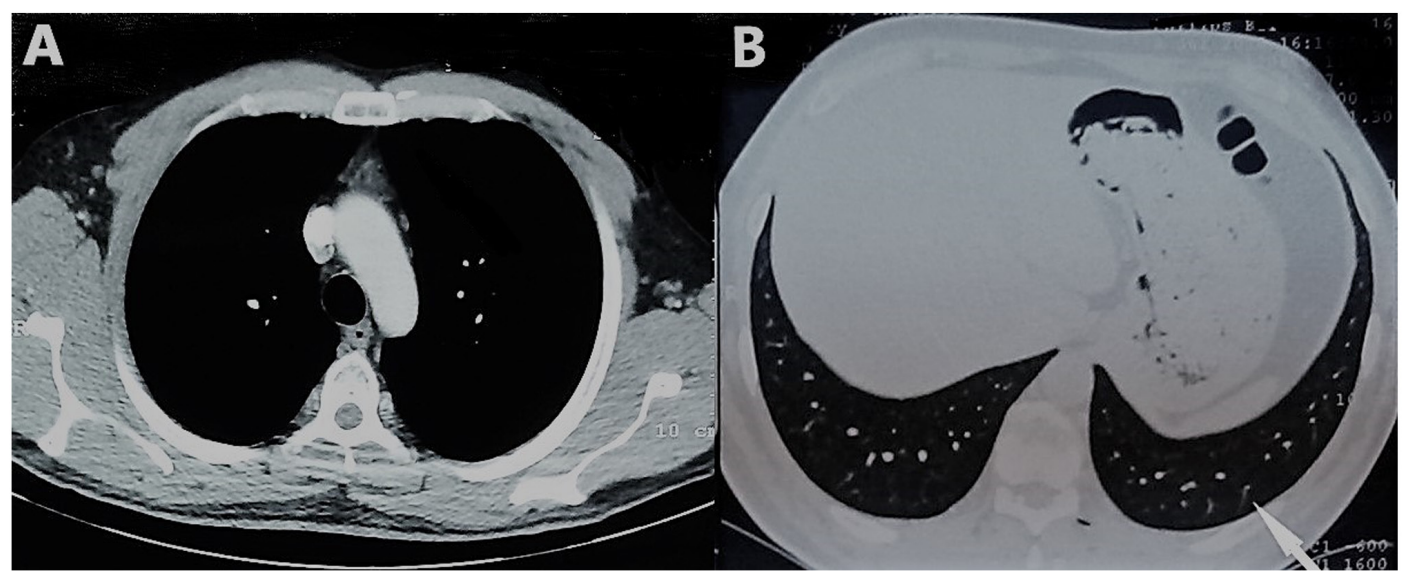

Figure 5. Chest computed tomography after 6 weeks of treatment (A) Chest computed tomography (mediastinal window). (B) Chest computed tomography (lung window). Complete remission of the lesions can be seen in both the panels.

complicated with anterior mediastinal abscess through hematogenous spread. Mediastinitis usually results from a wide range of etiologies, including esophageal perforation, lung infection, infected lymph nodes of the neck, an infected sternotomy wound, odontogenic infection, peritonsillar or retropharyngeal abscess, trauma of the cervix, epiglottitis, sinusitis and intravenous drug use (9). Mediastinitis as a result of hematogenous spread was also described by Calvano et al (3) in a case of an immunocompromised 47-year-old female who presented with pneumonia complicated with bacteremia and sepsis, and who developed multiple neck and mediastinal abscesses due to Staphylococcus aureus. Chang et al (2) reported a case of mediastinitis in a hemodialysis patient as a complication of Staphylococcus bacteremia, while Er et al (10) described a case of central venous catheter-associated mediastinitis due to Candida species.

In addition, mediastinitis as a complication of primary skin infections is also a rare entity. Brisset et al (1) reported a case of Staphylococcus aureus mediastinitis in an immunocompetent adult following back skin abscess. To the best of our knowledge, the present case is the second report of such a case of mediastinitis secondary to hematogenous spread following skin infection.

Treatment of acute mediastinitis is based on administration of antibiotics and control of the source of inflammation with surgical debridement (11). Mediastinitis, developing as a postoperative complication, usually requires rapid surgical debridement. Generally, management with multiple surgical procedures is needed in $\sim 30 \%$ of patients. The surgical approach depends on the extent of the disease and structures involved (12). The usual duration of therapy for mediastinitis is up to 4-6 weeks depending on the extent of surgical debridement performed (13).

In conclusion, development of mediastinitis, with hematogenous spread following skin infection, is a rare clinical entity. With the increase of described MRSA infectious disease cases, clinicians should remain alert for future cases of MRSA mediastinitis and consider mediastinitis in the differential diagnosis when a patient presents with respiratory symptoms, such as thoracic pain and dyspnea, as well as signs of skin infection.

\section{Acknowledgements}

Not applicable.

\section{Funding}

No funding was received.

\section{Availability of data and materials}

The datasets used and/or analyzed during the present study are available from the corresponding author on reasonable request.

\section{Authors' contributions}

VEG, KM and DM conceptualized the study. AGk, PP, PD, NG, NT and XT made substantial contributions to the acquisition, analysis and interpretation of the data. CD and $\mathrm{AGa}$ prepared the figures. All authors contributed to manuscript revision and approved the final manuscript. PS and SC confirm the authenticity of all the raw data.

\section{Ethics approval and consent to participate}

Not applicable.

\section{Patient consent for publication.}

Written informed consent was obtained from the patient for publication of this case report and any accompanying images.

\section{Competing interests}

The authors declare that they have no competing interests.

\section{References}

1. Brisset J, Daix T, Tricard J, Evrard B, Vignon P, Barraud O and François B: Spontaneous community-acquired PVL-producing Staphylococcus aureus mediastinitis in an immunocompetent adult - a case report. BMC Infect Dis 20: 354, 2020. 
2. Chang CH,Huang JY,Lai PC and Yang CW: Posterior mediastinal abscess in a hemodialysis patient - a rare but life-threatening complication of Staphylococcus bacteremia. Clin Nephrol 71: 92-95, 2009

3. Calvano TP,Ferraro DM,Prakash V, Mende K and Hospenthal DR: Community-associated methicillin-resistant Staphylococcus aureus mediastinitis. J Clin Microbiol 47: 3367-3369, 2009.

4. Gajdács M: The continuing threat of methicillin-resistant Staphylococcus aureus. Antibiotics (Basel) 8: 52, 2019.

5. Stefani S and Goglio A: Methicillin-resistant Staphylococcus aureus: Related infections and antibiotic resistance. Int J Infect Dis 14 (Suppl 4): S19-S22, 2010.

6. Jabłoński S, Brocki M, Krzysztof K, Wawrzycki M, Santorek-Strumiłło E, Łobos M and Kozakiewicz M: Evaluation of prognostic value of selected biochemical markers in surgically treated patients with acute mediastinitis. Med Sci Monit 18: CR308-CR315, 2012.

7. Kimura A, Miyamoto S and Yamashita T: Clinical predictors of descending necrotizing mediastinitis after deep neck infections. Laryngoscope 130: E567-E572, 2020.

8. Smith A and Sinzobahamvya N: Anterior mediastinal abscess complicating septic arthritis. J Pediatr Surg 27: 101-102, 1992.
9. Lin YY, Hsu CW, Chu SJ, Chen SC and Tsai SH: Rapidly propagating descending necrotizing mediastinitis as a consequence of intravenous drug use. Am J Med Sci 334: 499-502, 2007.

10. Er F, Nia AM, Caglayan E and Gassanov N: Mediastinitis as a complication of central venous catheterization. Infection 38: 509, 2010.

11. Abu-Omar Y, Kocher GJ, Bosco P, Barbero C, Waller D, Gudbjartsson T, Sousa-Uva M,LichtPB,Dunning J,Schmid RA, et al: European Association for Cardio-Thoracic Surgery expert consensus statement on the prevention and management of mediastinitis. Eur J Cardiothorac Surg 51: 10-29, 2017.

12. Prado-Calleros HM, Jiménez-Fuentes E and Jiménez-Escobar I: Descending necrotizing mediastinitis: Systematic review on its treatment in the last 6 years, 75 years after its description. Head Neck 38 (Suppl 1): E2275-E2283, 2016.

13. El Oakley RM and Wright JE: Postoperative mediastinitis: Classification and management. Ann Thorac Surg 61: 1030-1036, 1996.

This work is licensed under a Creative Commons Attribution-NonCommercial-NoDerivatives 4.0 International (CC BY-NC-ND 4.0) License. 